\title{
Context dependent learning in the serial RT task
}

\author{
Elger L. Abrahamse $\cdot$ Willem B. Verwey
}

Received: 2 May 2006 / Accepted: 5 July 2007 / Published online: 3 August 2007

(C) Springer-Verlag 2007

\begin{abstract}
This study investigated the development of contextual dependencies for sequential perceptual-motor learning on static features in the learning environment. In three experiments we assessed the effect of manipulating task irrelevant static context features in a serial reaction-time task. Experiment 1 demonstrated impaired performance after simultaneously changing display color, placeholder shape, and placeholder location. Experiment 2 showed that this effect was mainly caused by changing placeholder shape. Finally, Experiment 3 indicated that changing context affected both the application of sequence knowledge and the selection of individual responses. It is proposed either that incidental stimulus features are integrated with a global sequence representation, or that the changed context causes participants to strategically inhibit sequence skills.
\end{abstract}

\section{Introduction}

Research on verbal memory tasks has revealed better retrieval performance if the original learning context is reinstated during test administration (Smith \& Vela, 2001). A variety of context stimuli have been shown to reduce performance when changed, including background music (Smith, 1985), physiological state (Eich, 1980), and general physical environment (Godden \& Baddeley, 1975; Eich, 1985). Wright and Shea (1991) extended the examination of the effects of task irrelevant context on verbal memory

E. L. Abrahamse $(\bowtie) \cdot$ W. B. Verwey

Cognitive Psychology and Ergonomics,

Faculty of Behavioral Sciences,

Universiteit Twente, Postbus 217,

7500 AE Enschede, The Netherlands

e-mail: e.l.abrahamse@gw.utwente.nl performance to the reproduction of perceptual-motor responses. They proposed a model in which they discriminate between stimuli that are explicitly identified as essential to task performance (intentional) and those that are not (incidental). In their study participants practiced three keying sequences, with numbers to indicate the elements of each sequence. Each sequence was consistently paired during practice with a combination of a particular display color, a specific tone, a certain position on the screen, and a particular placeholder shape. Subsequently changing these incidental stimuli impaired key pressing performance. This finding was interpreted as support for the notion that motor skills can be context dependent.

However, because the incidental stimuli consistently covaried with the intentional stimulus, associative learning instead of a general context effect might explain the effects reported by Wright and Shea (1991). It can be argued that through their strong temporal relationship with the intentional stimulus, the incidental stimuli became more or less intentional with practice. We propose that incidental context features should be further subdivided into those that co-vary with the intentional stimulus, and those that are continuously present during training, independently of the presence of intentional stimuli (static features). The purpose of the present study is to examine the potential contextual dependency of motor skill learning on static context features.

To our knowledge context-dependence has not been investigated before with the serial reaction-time (SRT) task (Nissen \& Bullemer, 1987). In this task participants are required to respond as fast and accurately as possible to the location of successively presented stimuli. Unbeknownst to the participants, however, the stimuli follow a specific order. With practice, reaction times (RTs) turn out to decrease. To make sure that that improvement is not a general effect of practice, a random or pseudo-random 
block of stimuli is presented at the end of the practice phase. The increase in RTs and/or errors in this random block relative to the final sequence blocks serves as an index for sequence learning. As participants are often not able to tell that the stimuli followed a particular order after the experiment, the task is said to involve implicit sequence learning. The SRT task has become one of the major paradigms for studying implicit learning (for reviews see e.g. Keele, Ivry, Hazeltine, Mayr \& Heuer, 2003; Stadler \& Frensch, 1998).

We suspect that dependencies on static context are most pregnant for implicitly learned perceptual-motor skills as the effects of implicit sequence learning are mostly described as more vulnerable to changes in its triggering conditions (Jiménez, Vaquero, \& Lupiáñez, 2006), and "tend to be less manipulable and more context bound" (Berry \& Dienes, 1993, p. 13; but see Willingham, 1997, for critical commentaries on the difference in flexibility between implicit and explicit memory). Because implicit learning is said to be highly stimulus-driven it may be directly affected by changes of stimulus input (even if task irrelevant). Designed to study implicit learning, the SRT task may thus be a promising candidate for revealing context dependent motor skills. We tried to reduce explicit learning by using a response-tostimulus interval (RSI) of $0 \mathrm{~ms}$ in the current experiments (see Destrebecqz \& Cleeremans, 2001).

\section{Experiment 1}

In Experiment 1 participants responded to the onset of stimuli presented in a fixed order in a typical serial RT task. After they practiced this sequence in a specific context (with a particular placeholder location, display color, and placeholder shape), we changed these context features. We hypothesized that if certain features of the incidental context are stored in memory along with response events, in this case while performing a SRT, then changing these features would impair performance.

\section{Method}

\section{Participants}

Sixteen students at the University of Twente participated in exchange for course credits. They were aged between 18 and 30 , had no hand or vision problems, and were naïve as to the purpose of the study.

\section{Apparatus and setting}

Stimulus presentation, timing, and data collection was achieved using the E-prime $(1.1$ experimental software package on a standard Pentium $₫$ IV class PC. Stimuli were presented on a 17 inch Philips 107T5 display running at $1,024 \times 768$ pixel resolution in 32 bit color, and refreshing at $85 \mathrm{~Hz}$. The viewing distance was approximately $50 \mathrm{~cm}$, but not strictly controlled.

\section{Procedure}

The task consisted of a typical SRT task (Nissen \& Bullemer, 1987), and involved twenty blocks of trials. The experiment started with two random blocks, in which stimulus position did not follow a particular pattern, to prevent participants from discovering the pattern in an initial attempt. These blocks were followed by 16 sequence training blocks, a transfer block, and finally one more sequence training block. Each of these blocks started with four random trials and was followed by nine repetitions of a 12-element sequence. Participants were instructed to respond as fast and accurately as possible, using the middle and index finger of both hands to press the $c, v, b$, and $n$ keys on the keyboard. A correct response was defined as the participant pressing the appropriate key within a 2-s time limit. Erroneous responses were signalled to the participants, after which the next stimulus was presented after a 2 -s interval. This relatively long interval was intended to motivate the participant to prevent errors. Short $1 \mathrm{~min}$ breaks were provided in between blocks. The sequence consisted of second order conditional (SOC) transitions: 121342314324 (Reed \& Johnson, 1994). In a SOC sequence all elements and pairs of elements occur equally frequent. Consequently, performance cannot improve from just learning that certain elements or element pairs occur more often than others.

Each display provided both intentional and incidental stimuli. The intentional stimulus consisted of filling one of the four horizontally aligned placeholders with red. The incidental stimuli consisted of the color of the screen background, the placeholder location, and the shape of the placeholders. In Context A we used a white display, with four rectangular placeholders at the top of the screen. Context B involved a dark grey display, with four triangular placeholders placed at the bottom of the screen. From a viewing distance of $50 \mathrm{~cm}$, stimulus angle measured $2.3^{\circ} \times 2.0^{\circ}$ for the rectangles, and $2.3^{\circ} \times 2.7^{\circ}$ for the triangles. To make the distance between the placeholders identical in contexts A and B, the triangles were clustered with the first and third triangle pointing upwards, and the second and fourth triangle pointing downwards. Training and testing with either Context A or Context B was counterbalanced across participants: half of the participants trained with Context A, and encountered Context B during transfer at Block 19, while the other participants trained with Context B and were tested with Context A. Just before the context was changed in Block 19, participants were informed 
that some changes would occur on the screen, but that in other respects the task would remain the same.

Finally, participants performed a free generation task to examine the extent to which they were aware of the order of the sequence elements. This involved telling them that there had been a 12-element fixed order, and then having them write down the complete 12-element sequence that according to them had been repeated during the experiment (Witt \& Willingham, 2006).

Results

\section{Reaction-time task}

RT analyses excluded erroneous key presses, and RTs exceeding the criterion of mean plus 3 standard deviations. This eliminated less than $5 \%$ of the data in the acquisition and the test phase. Also, the four random trials at the beginning of each block were excluded from analysis. Mean reaction times and accuracy scores were calculated for each block, for each participant.

Practice phase Figure 1 shows the mean RT for each block. We performed a repeated measure ANOVA on reaction times with Block (18; Blocks 1 to 18) as a within-subject variable. This analysis revealed a significant effect for Block, $F(17,255)=59.0, P<0.0001$, indicating improvement with practice.

Test phase Rather than changing the order of the elements, as is typical in the SRT task, we changed the context in Block 19. The effect was tested with another repeated

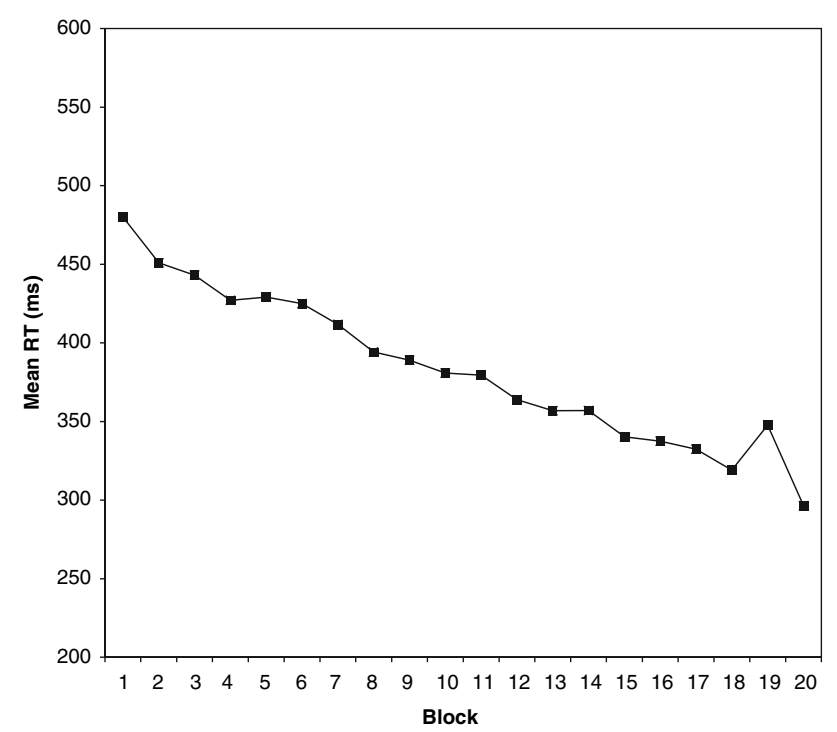

Fig. 1 Mean RT for Blocks 1-20 in Experiment 1. In Block 19 display color, placeholder shape, and placeholder location were simultaneously changed measures ANOVA on reaction times with Block (2; mean of Blocks 18 and 20, versus Block 19) as within-subject variable. As depicted in Fig. 1, reactions were slower in Block 19, $F(1,15)=39.0, P<0.0001$, confirming that performance decreased with context change.

Finally, to perform an error analysis, we used an arcsin transformation (Winer, Brown \& Michels, 1991) to stabilize variances. Then a repeated measures ANOVA was run on these transformed error scores for the practice phase with Block (18) as a within-subject variable. This showed no significant results. A similar analysis on the mean error percentages of Blocks 18 and 20 versus Block 19 did not reveal reliable differences either. Error percentages were around $2.5 \%$ for Block 18, 19 and 20.

\section{Awareness}

An awareness score was calculated by counting the number of correctly generated 3-element chunks in the free generation task, and dividing this number by 12 , as participants could generate a maximum of twelve correct chunks. The awareness score $($ mean $=0.36)$ was compared to chance level (which is 0.33 as no repetitions were allowed) of generating correct sequence chunks with a one-sample $t$ test (Destrebecqz \& Cleeremans, 2001). This indicated that across the entire group the mean awareness score did not differ reliably from chance level, $t(15)=0.6, P=0.5$. Inspection of individual awareness scores indicated that awareness varied amongst the participants. However, grouping participants according to their awareness scores (low versus high awereness) and including this as an independent variable in the above ANOVAs did not produce reliable awareness effects, and these analyses have therefore not been reported.

\section{Discussion}

The purpose of Experiment 1 was to test the hypothesis that sequential skills as assessed with the serial RT task may become dependent on the context they have been acquired in, even if this context remains fixed during training. If so, performance should be impaired when the training context is changed. The results from Experiment 1 support this idea for the combined effect of changing display color, placeholder location and placeholder shape.

\section{Experiment 2}

Experiment 1 indicates that changing seemingly task irrelevant features can impair performance. The question remains, though, whether all three incidental context features had been equally effective. Experiment 2 was 
conducted to assess the separate contributions of each individual context feature used in Experiment 1.

Method

\section{Participants}

Forty-eight first year bachelor students at the University of Twente participated in exchange for course credits. They were aged between 18 and 30, had no vision or arm problems, and were naïve as to the purpose of the study.

\section{Apparatus and setting}

Apparatus and setting were the same as in Experiment 1.

\section{Procedure}

The procedure was the same as for Experiment 1, except now there were three groups of participants. In one group the effect of display color (white versus gray) was tested, in the second group the effect of the placeholder shape (rectangular versus triangular) was tested, and in the third group the effect of the location of the four placeholders on the computer screen (top versus bottom) was tested. The feature combinations actually used are summarized in Table 1 . The use of either Context A or B for practice in a particular context group was counterbalanced across participants. Participants were randomly assigned to groups.

Results

\section{Reaction-time task}

RT analyses excluded erroneous key presses, and RTs exceeding a criterion of mean plus 3 standard deviations. The latter requirement eliminated less than $5 \%$ of the data

Table 1 Contexts A and B as used with the three experimental groups of Experiment 2

\begin{tabular}{llll}
\hline $\begin{array}{l}\text { Experimental } \\
\text { group }\end{array}$ & Feature & Context A & Context B \\
\hline Display color & Display color & White & Grey \\
& Placeholder shape & Rectangular & Rectangular \\
& Placeholder location & Middle & Middle \\
Placeholder & Display color & White & White \\
shape & Placeholder shape & Rectangular & Triangular \\
& Placeholder location & Middle & Middle \\
Placeholder & Display color & White & White \\
location & Placeholder shape & Rectangular & Rectangular \\
& Placeholder location & Top & Bottom \\
\hline
\end{tabular}

across the acquisition and test phases. Also, the four random trials at the beginning of each block were excluded from analysis. Mean reaction times and accuracy scores were calculated for each block, for each participant.

Practice phase A Group (3; color, shape, location) $\times$ Block (18) repeated measures ANOVA on reaction times was performed with Block as within-subject variable. This showed an effect for Block, $F(17,765)=102.5, P<0.0001$, but no significant Group main effect, $F(2,45)=1.8, P=0.15$, or Block by Group interaction, $F(34,765)=0.6, P=0.8$. This indicates that learning did not differ across groups (Fig. 2). The same analysis was performed on transformed error scores, but this revealed no reliable differences at all $(F \mathrm{~s}<1.2, P \mathrm{~s}>0.25)$.

Test phase Another Group (3; color, shape, location) $\times$ Block (2; mean of Blocks 18 and 20 versus Block 19) ANOVA on reaction times was performed with Block as within-subject variable. This resulted in a significant Block effect, $F(1,45)=12.4, P<0.005$, and a Block by Group interaction, $F(2,45)=18.6, P<0.0001$. Separate pairedsample $t$ tests on Block 19 versus the mean of Blocks 18 and 20 were carried on for all three groups. Bonferroni correction yielded an $\alpha$ of 0.013 . This resulted in a significant effect for the placeholder shape group, $t(15)=4.7$, $P<0.0001$, but not for the placeholder location group, $t(15)=2.5, P=0.03$, or the display color group, $t(15)=2.1$, $P=0.05$. So, performance was significantly impaired after changing the placeholder shape in the test phase while changing the location on the screen or changing the display color failed to produce a significant effect.

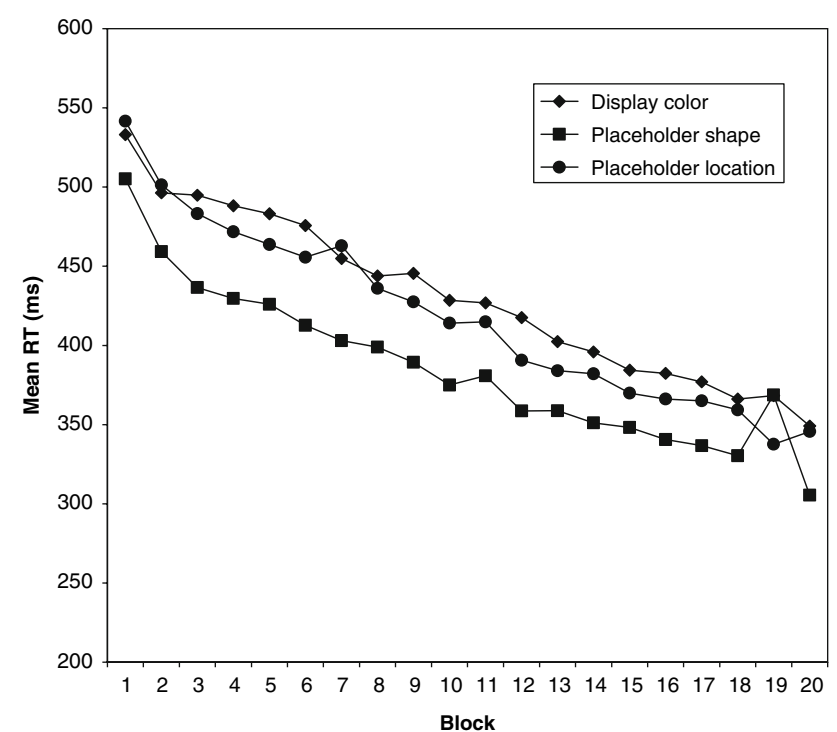

Fig. 2 Mean RT per experimental group for Blocks 1-20 in Experiment 2. In Block 19 for each group another context feature was changed 
Finally, a repeated measures ANOVA on arcsine-transformed error scores was performed for the means of Blocks 18 and 20 in comparison with those of Block 19. This revealed a significant Block by Group interaction, $F(2,45)=5.2, \quad P<0.01$. Paired-sample $t$ tests for each group ( $\alpha=0.013$ after Bonferroni correction) showed a significant Block effect only for the placeholder shape group, $t(15)=4.0, P<0.005$, with error percentages amounting to 3.3, 3.9 and 2.9\% for Blocks 18-20. The other two were far from significant $(t \mathrm{~s}<0.8, P \mathrm{~s}>0.4$; error percentages were always below $4 \%)$.

\section{Awareness}

Awareness was calculated in the same way as in Experiment 1. A one-way ANOVA was performed on awareness scores with Group as between-subject variable (mean awareness scores were $0.35,0.33$, and 0.33 for the display color, placeholder shape, and placeholder location groups, respectively). This indicated no reliable differences between groups, $F(2,45)=0.06, P=0.9$. Then the difference between the awareness score and chance level was tested for each group separately. This indicated no reliable differences ( $t \mathrm{~s}<0.5, P \mathrm{~s}>0.6)$, thus again this indicates that learning was mainly implicit. Again, inspection of individual awareness scores showed that some participants had some awareness. Therefore, we also performed analyses with low and high awareness as independent variable. This did not produce any reliable effects, while keeping the relevant findings of this experiment intact.

\section{Discussion}

Experiment 2 suggests that placeholder shape had produced almost the entire context effect in Experiment 1, even though display color may have contributed, too. Participants were less able to efficiently apply their sequence knowledge when placeholder shape had been changed: During the test block, they showed significantly increased response latencies, and produced more errors. This indicates that performance had become dependent on the taskirrelevant shape of the stimulus, and not significantly so on display color and placeholder location.

\section{Experiment 3}

The results of Experiments 1 and 2 demonstrate that performance in the serial RT task became context dependent in the course of practice. However, in the test block of those experiments we manipulated just the context and not the order of the individual elements (i.e. the sequence). As responding to individual stimuli may well continue to be used in the serial RT task we are not yet able to determine whether the context change affected sequencing skill or response selection skill. Experiment 3 was aimed at testing whether the context effect we obtained in Experiments 1 and 2 was associated with response selection (which should affect random and fixed sequences, but random more than fixed), with sequencing skill (which should influence just fixed sequences), or with both (which should affect fixed sequences more than random). To that end, we had a group of participants perform in an experiment that was identical to the placeholder shape condition of Experiment 2, but in which there was no fixed sequence. We then compared the obtained results with those of the placeholder group in Experiment 2.

\section{Method}

\section{Participants}

Sixteen first year bachelor students at the University of Twente participated in exchange for course credits. They were aged between 18 and 30, had no vision or arm problems, and were naïve as to the purpose of the study.

\section{Apparatus and setting}

Apparatus and setting were identical to those in Experiments 1 and 2 .

\section{Procedure}

The procedure was identical to that of the placeholder shape group in Experiment 2, except that this time the stimuli were ordered in a pseudo-random way. The pseudo-random blocks in this experiment consisted of nine different SOC sequences that were randomly picked from a pool of twelve, with no element and sequence repetitions allowed. This procedure was repeated for every next random block.

\section{Results}

All RT analyses excluded erroneous key presses, and RTs exceeding a criterion of mean plus 3 standard deviations. The latter requirement eliminated less than $5 \%$ of the data. Mean reaction times and accuracy scores were calculated for each block and for each participant. Because the procedures in Experiments 2 and 3 were identical, the analyses used the data from the placeholder shape group of Experiment 2 .

Practice phase RTs obtained in Experiment 3 are depicted in Fig. 3 along with those assessed with the placeholder shape group of Experiment 2. An Order (2; random 


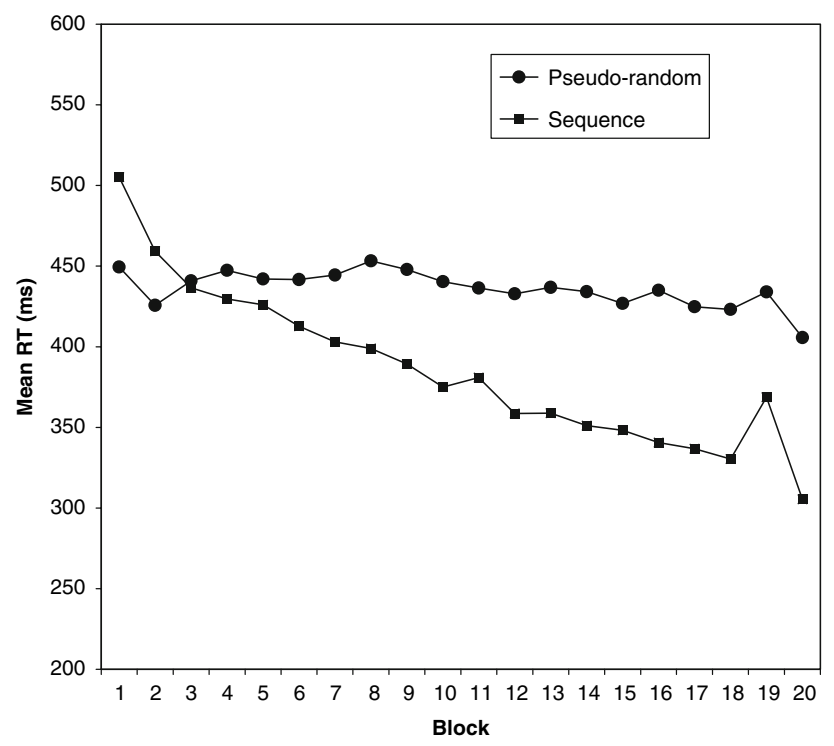

Fig. 3 Mean RTs for the group practicing with a pseudo-random sequence (Experiment 3 ) and the group practicing with a fixed sequence (taken from Experiment 2). In both groups, Block 19 involved changing placeholder shape

versus sequential) $\times$ Block (18) repeated measures ANOVA on reaction times was performed with Block as within-subject variable. This showed significant main effects for both Block, $F(17,510)=30.5, P<0.0001$, and Order, $F(1,30)=6.7, P<0.05$, and a significant Block by Order interaction, $F(17,510)=20.4, \quad P<0.0001$. As expected, this indicates that participants in the random SRT condition showed less improvement with practice than the participants in the normal SRT (see Fig. 3).

The same analysis was performed on transformed error scores. This produced no significant results, even though Block almost reached significance, $F(17,510)=1.8$, $P=0.07$, indicating a trend towards less errors with practice (error percentages were always below 5\%).

Test phase An Order (2; random versus sequential) $\times$ Block (2; mean of Blocks 18 and 20 versus Block 19) repeated measures ANOVA on reaction times was performed with Block as within-subject variable. This produced significant main effects for both Block, $F(1,30)=26.9, P<0.0001$, and Order, $F(1,30)=16.2, P<0.0001$, and a significant Block by Order interaction, $F(1,30)=5.2, P<0.05$. This interaction demonstrates that context had a stronger effect in the sequence group of Experiment 2 than in the random group of Experiment 3. Experiment 2 already reported that changing placeholder shape significantly increased RT, $t(15)=4.7, P<0.0001$. We then performed a paired-sample $t$ test to determine whether changing placeholder shape had reduced performance in Block 19 for the random group too. This appeared to be the case, $t(15)=2.4$,
$P<0.05$. Taken together, these results show that changing the placeholder shape has a dominant effect on applying sequence knowledge, but that response selection skill was affected too.

A similar repeated measures ANOVA was performed on arcsin transformed error percentages. This revealed a significant main effect for Block, $F(1,30)=13.6, P<0.005$, but no Block by Order interaction, $F(1,30)=2.4, P=0.1$. Error percentages amounted to 2.7 and $3.9 \%$ for the mean of Block 18 and 19, and Block 20, respectively.

\section{Discussion}

In Experiment 2 we showed that the impaired performance in Experiment 1 after changing the context was mainly caused by changing placeholder shape. However, it remained unclear whether this feature affected sequencing skill, or perhaps some residual selection of responses on basis of the stimulus, that is assumed to continue with implicitly learned sequences. Experiment 3 clearly shows that it was primarily the application of sequence knowledge that was affected by this context change, as this manipulation disrupted performance more in the normal SRT condition than the random SRT condition. In line with earlier conclusions (Shea \& Wright, 1995), Experiment 3 demonstrates also that response selection had become a skill that is affected by this context change.

\section{General discussion}

The main purpose of this study was to determine whether sequential movement skills as obtained in the serial RT task may become susceptible to changes in the context, as has been demonstrated with various memory tasks (e.g., Smith \& Vela, 2001). We manipulated only static context features. Our results indicate that, in addition to response selection skill (Shea \& Wright, 1995), sequential skill in the SRT task becomes susceptible to the task irrelevant shape of the placeholder that contained the imperative stimuli, though there was a trend in Experiment 2 that changing background colour had a detrimental effect too. Changing the placeholder location revealed a trend towards better performance at the test block. This latter effect may well be a motivational effect: any change will trigger renewed attention to the task at hand.

The current findings show that the serial RT task is a useful paradigm for exploring contextual dependent motor skill acquisition. However, the mechanism underlying the influence of incidental perceptual features on sequence performance remains largely unclear. Below we will elaborate on two general alternatives to account for the current findings. The first is derived from the notion that stimulus 
features are becoming part of a global sequence skill representation. Second, the change in context could have affected performance in a less direct manner, as it may have brought participants to inhibit existing sequence skills.

A number of different levels of serial learning have been proposed by various authors. From the perceptual learning view, sequence learning is predominantly based on associations between successive stimuli (stimulus-to-stimulus or S-S learning). In contrast, the motor learning account states that associations are formed mainly between successive responses (response-to-response or R-R learning). Additionally, other studies support sequence learning on intermediate levels of information processing (e.g. Deroost \& Soetens, 2006), or as a kind of response effect learning (response-to-stimulus or R-S learning; Ziessler \& Nattkemper, 2001). There is growing consensus that sequence learning is predominantly response based, as learning on the motor level is supported by many behavioural (Nattkemper \& Prinz, 1997; Willingham, 1999) and neuropsychological (e.g. Bischoff-Grethe, Goedert, Willingham \& Grafton, 2004; Grafton, Hazeltine \& Ivry, 1995) studies. The role of perceptual learning on the other hand is still heavily debated. In line with other studies (e.g. Abrahamse, Van der Lubbe \& Verwey, 2007; Remillard, 2003; Mayr, 1996), the current study adds support to the notion that sequence learning in a SRT task is not completely motor based (i.e. independent of the stimuli), as changing a stimulus feature that was not directly relevant to the task (i.e. the placeholder shape) had a clear effect on performance. The representation underlying sequence skill, then, may include incidental stimulus information (either through $\mathrm{S}-\mathrm{S}$ associations, $\mathrm{R}-\mathrm{S}$ associations, or learning at intermediate levels of information processing), implying that when these features are changed, the skill representation can no longer be as easily retrieved from memory (maybe under certain conditions incidental features of the rest of the task environment are also included, as display color almost produced a reliable effect). This is in line with the notion that sequence learning is partly produced by an automatic associative process (Jiménez \& Méndez, 1999), on the condition that it involves only that information that has been selected for processing (e.g. Frensch \& Miner, 1994), including some features that may not be crucial to task execution. The role of co-activation in memory systems to account for automatic associations is fundamental to many learning theories (e.g. Logan, 1988). Future research will be needed to explore the role of selective attention for the development of context dependability of sequential skills in more detail.

A second explanation may be worth mentioning here. Above we propose a rather direct influence of incidental stimulus features on sequence performance, as they may have been integrated in a global sequence representation. However, changing the perceptual stimulus features may have also influenced performance in a less direct manner, that is, through an increased tendency for more direct control by the participants (Luis Jiménez, personal communication). The idea is that when features of the task or task environment change, participants may no longer (trust to) rely on their implicit skills (see also Schneider \& Fisk, 1982; Moore \& Stevenson, 1994). Rather, they are strategically evaluating the task and its environment, while inhibiting automatic processes. So, the present context effect could be caused also by strategic inhibition of sequencing skill, rather than by difficulty in memory retrieval.

In conclusion, changing the context (i.e. the placeholder shape, and possibly also the display color) has a clear effect on sequencing skill. This may have been caused by the impeded retrieval of a global sequence representation from memory (implying that sequence learning in the SRT task is not predominantly motor-based), or by strategic inhibition of sequencing skills. Further research is needed to explore the mechanism underlying the present findings in more detail.

Acknowledgments We would like to thank Jan-Henk Annema for his assistance with the data collection and statistical analyses.

\section{References}

Abrahamse, E. L., Van der Lubbe, R. H.J., \& Verwey, W. B. (2007). Asymmetry in learning between a tactile and visual serial RT task. The Quarterly Journal of Experimental Psychology (in press).

Berry, D. C., \& Dienes, Z. (1993). Implicit learning: Theoretical and empirical issues. Hove: Erlbaum.

Bischoff-Grethe, A., Goedert, K. M., Willingham, D. B., \& Grafton, S. T. (2004). Neural substrates of response-based sequence learning using fMRI. Journal of Cognitive Neuroscience, 16, 127-138.

Deroost, N., \& Soetens, E. (2006b). The role of response selection in sequence learning. The Quarterly Journal of Experimental Psychology, 59, 449-456.

Destrebecqz, A., \& Cleeremans, A. (2001). Can sequence learning be implicit? New evidence with the Process Dissociation Procedure. Psychonomic Bulletin \& Review, 8, 343-350.

Eich, J. E. (1980). The cue-dependent nature of state-dependent retrieval. Memory \& Cognition, 8, 152-173.

Eich, J. E. (1985). Context, memory, and integrated item/context imagery. Journal of Experimental Psychology: Learning. Memory, \& Cognition, 11, 764-770.

Frensch, P. A., \& Miner, C. S. (1994). Effects of presentation rate and individual differences in short-term memory capacity on an indirect measure of serial learning. Memory \& Cognition, 22, 95-110.

Godden, D. R., \& Baddeley, A. D. (1975). Context-dependent memory in two natural environments: on land and underwater. British Journal of Psychology, 66, 325-331.

Grafton, S. T., Hazeltine, E., \& Ivry, R. (1995). Functional anatomy of sequence learning in normal humans. Journal of Cognitive Neuroscience, 7, 497-510.

Jiménez, L., \& Méndez, C. (1999). Which attention is needed for implicit sequence learning? Journal of Experimental Psychology: Learning, Memory and Cognition, 25, 236-259.

Jiménez, L., Vaquero, J. M. M., \& Lupiáñez, J. (2006). Qualitative differences between implicit and explicit sequence learning. Journal 
of Experimental Psychology: Learning, Memory, and Cognition, 32, 475-490.

Keele, S. W., Ivry, R. B., Hazeltine, E., Mayr, U., \& Heuer, H. (2003). The cognitive and neural architecture of sequence representations. Psychological Review, 110, 316-339.

Logan, G. D. (1988). Towards an instant theory of automatization. Psychological Review, 95, 492-527.

Mayr, U. (1996). Spatial attention and implicit sequence learning: Evidence for independent learning of spatial and nonspatial sequences. Journal of Experimental Psychology: Learning, Memory and Cognition, 22, 350-364.

Moore, W. E., \& Stevenson, J. R. (1994). Training for trust in sport skills. The Sport Psychologist, 8, 1-12.

Nattkemper, D., \& Prinz, W. (1997). Stimulus and response anticipation in a serial reaction task. Psychological Research, 60, 98-112.

Nissen, M. J., \& Bullemer, P. (1987). Attentional requirements of learning: Evidence from performance measures. Cognitive Psychology, 19, 1-32.

Remillard, G. (2003). Pure perceptual-based sequence learning. Journal of Experimental Psychology: Learning, Memory and Cognition, 29, 518-597.

Schneider, W., \& Fisk, A. D. (1982). Concurrent automatic and controlled visual search: Can processing occur without resource cost? Journal of Experimental Psychology: Learning, Memory and Cognition, 8, 261-278.
Shea, C. H., \& Wright, D. L. (1995). Contextual dependencies: Influence on response latency. Memory, 3, 81-95.

Smith, S. M. (1985). Background music and context-dependent memory. American Journal of Psychology, 98, 591-603.

Smith, S. M., \& Vela, E. (2001). Environmental context-dependent memory: A review and meta-analysis. Psychonomic Bulletin \& Review, 8, 203-220.

Stadler, M. A., \& Frensch, P. A. (1998). Handbook of implicit learning. Thousand Oaks: Sage.

Willingham, D. B. (1997). Commentary. Implicit and explicit memory do not differ in flexibility: Comment on Dienes and Berry (1997). Psychonomic Bulletin \& Review, 4, 587-591.

Willingham, D. B. (1999). Implicit motor sequence learning is not purely perceptual. Memory and Cognition, 27, 561-572.

Winer, B. J., Brown, D. R., \& Michels, K. M. (1991). Statistical principles in experimental design, 3rd edn. New York: McGraw-Hill.

Witt, J. K., \& Willingham, D. T. (2006). Evidence for separate representations for action and location in implicit motor sequencing. Psychonomic Bulletin \& Review, 13, 902-907.

Wright, D. L., \& Shea, C. H. (1991). Contextual dependencies in motor skills. Memory \& Cognition, 19, 361-370.

Ziessler, M., \& Nattkemper, P. (2001). Learning of event sequences is based on response-effect learning: Further evidence from a serial reaction time task. Journal of Experimental Psychology: Learning, Memory and Cognition, 27, 595-613. 